\title{
Aporías del proceso refundacional chileno
}

\section{Aporias in the Refoundation of Chile}

Alberto Mayol Miranda ${ }^{1}$ Felipe Vidal Muñoz ${ }^{2}$

Cómo citar este artículo: Mayol Miranda, A., Vidal Muñoz, F. (2021). Aporías del proceso refundacional chileno. Revista de Ciencias Sociales Ambos Mundos, (2), 37-46. https://doi.org/10.14198/ambos.20990

\begin{abstract}
Resumen
El propósito de este artículo es caracterizar el escenario de transformación social en el Chile contemporáneo, comprendiendo al estallido social como un proceso que ha condensado energías sociales capaces de abrir espacio para una reestructuración del país. El texto consta de tres secciones. Primero, se argumenta que el estallido social que comenzó en Chile en el año 2019 puede ser visto como un acontecimiento refundacional, inaugurando un orden social que lucha por distanciarse y diferenciarse del orden anterior. En la segunda parte se describe la configuración y crisis de dicho orden anterior, engendrado durante la dictadura (1973-1990) y continuado durante la transición a la democracia (1991-2019). Finalmente, se discuten los desafíos y expectativas que existen en torno al proceso constituyente y la Convención Constitucional. Se concluye que, a pesar de haber puntos obvios (aumentar los derechos sociales, acabar con la discriminación institucionalizada, y asegurar una mayor participación ciudadana), no existe claridad con respecto a cómo una nueva Constitución podrá efectivamente procesar las demandas de cambio en Chile.
\end{abstract}

Palabras claves: Transformación social; estructura social; Chile; neoliberalismo; Constitución.

Abstract

The article aims to describe the current state of social change in contemporary Chile by interpreting the social oubreak as a process in which social energy is condensed, thus making possible a restructuring of the country. The text consists of three sections. First, it is argued that the social outbreak that began in Chile in 2019 can be understood as a re-founding event, ushering a new social order that struggles to differentiate itself from the previous one. Identifying its most relevant milestones between 1973 and 2019 , the second part describes the configuration and crisis of said previous order, generated during the dictatorship (1973-1990) and continued during the transition to democracy (1991-2019). Finally, the challenges and expectations around the constituent process and the Constitutional Convention are discussed. It is concluded that, despite obvious existing needs (increasing social rights, ending institutionalized discrimination, and ensuring greater citizen participation), there is no clarity regarding how a new Constitution could effectively respond to the claims for change in Chile.

Keywords: Social change; social structure; Chile; neoliberalism; Constitutions.

1. Alberto Mayol Miranda, Universidad de Santiago, Chile. alberto.mayol@usach.cl https://orcid.org/0000-0002-2562-3285

2. Felipe Vidal Muñoz, Universidad de Santiago, Chile. felipe.vidal.m@usach.cl https://orcid.org/0000-0002-2488-5435

Fecha recepción: 22/09/2021, Fecha aceptación: 21/10/2021

Licencia: Este trabajo está sujeto a una licencia de Reconocimiento 4.0 Internacional de Creative Commons (CC BY 4.0).

https://creativecommons.org/licenses/by/4.0/ 


\section{EL ESTALLIDO SOCIAL CHILENO COMO ACONTECIMIENTO REFUNDACIONAL}

La suspensión de un orden a partir de un suceso que es disruptivo respecto a ese orden podemos entenderla como un acontecimiento. El surgimiento posterior de un aparato normativo capaz de fundar un orden social, instalándose una lógica nueva y de carácter generativo, esto es, que puede reproducirse, complejizarse, y racionalizarse, se puede entender como un momento cero. Dicho de otro modo, en el primer instante, el acontecimiento es disruptivo y la materia se desacopla (las estructuras existentes pierden su forma); en el segundo es generativo, y la materia se acopla (nace una nueva estructura). Necesariamente un momento fundacional ha de surgir de una instancia que trasciende a la lógica anterior, es decir, desde fuera de las estructuras normativa, política y elitaria anteriores. Una refundación no es una reforma.

Vale la pena rescatar el trabajo del sociólogo Alberto Mayol (Mayol, 2012b, 2019, 2020), quien junto a su equipo ha trabajado durante años en torno a dos conceptos cuya articulación permite la comprensión de los momentos de crisis de todo orden social: energía y significado, nociones amplias, pero por lo mismo, con gran capacidad heurística. La energía es todo proceso que despliega alguna clase de fenómeno irritativo en la sociedad, que exige acciones asociadas, respuestas, reconstrucciones de sentido. Vale tanto para el arribo de una inversión de mil millones de dólares en un pequeño poblado (Araya et al., 2019), como para protestas enormes (Mayol Miranda \& Azócar Rosenkranz, 2011), fenómenos exógenos (climáticos, por ejemplo) (Jamison, 2010), una invasión extraterrestre o un profeta que señala ser Cristo (o, mejor dicho, que se ofrece como la segunda venida de Cristo) (Gaete Lagos, 2010; Gaitán Bayona, 2015; Tesche Roa, 2014). Todo proceso se hace social en la medida que los meros hechos se convierten en parte de la acción social y del significado del grupo (Weber, 1922/2014). Se vuelven inteligibles socioculturalmente. Esta cobertura suele tomar la forma de una narrativa. Todo excedente de energía requiere una forma cultural que la cubra y envuelva. Pero cuando se trata de un excedente de energía que supera cierto umbral, es decir, que se torna disruptivo, no basta con asimilarlo dentro de las categorías y significados existentes. 0 , más bien, éstos no dan abasto. Se necesita una nueva forma cultural para hacer legible a esa energía. Y eso suele conllevar una refundación del orden social y simbólico.
Argumentamos aquí que el estallido social chileno iniciado el año 2019 es un acontecimiento disruptivo, y que el proceso constituyente es el camino, todavía único y feble, a través del cual se intenta dar sentido a toda la energía liberada en el seno de la sociedad chilena. Es la forma en que se ha comenzado a cimentar una eventual refundación del orden social.

En rigor, la historia es más compleja. El primer acontecimiento ocurrió en 2011 y duró alrededor de un año en su proceso de desestructuración del orden existente, un orden que se puede denominar "transicional" en lo político o neoliberal en lo económico. En 2011 comenzó un ciclo de protestas que en origen se desplegó desde temas ambientales (proyecto Hidroaysén) (Latta, 2011; Reyes Herrera \& Rodríguez Torrent, 2015; Romero Toledo, 2014), luego migró a educación (donde llegó a su cénit con grandes movilizaciones por todo Chile) (Fleet, 2011), para finalmente mutar a exigencias regionalistas (Aysén, Calama, entre otros) y de salud pública (Freirina, Tocopilla, entre otros) (Delamaza et al., 2015; Penaglia Vásquez \& Valenzuela Van Treek, 2014; Valenzuela et al., 2016). El estallido social de 2019 aparece como un despliegue de energía disruptiva, un cataclismo, debido a una acumulación de esa energía que no fue procesada por el sistema político luego del álgido 2011.

Si el golpe de Estado de 1973 fue el acontecimiento originario que estableció aquel orden en Chile (impidiendo la realización del proyecto allendista), si podemos llamarlo un momento fundacional, entonces el estallido social de 2019 opera como "contragolpe", como respuesta y declaración del final de aquella creación en dictadura. Además, interpretamos al estallido como el hito que concluye el periodo histórico conocido como "la transición a la democracia" (hay quienes le llaman "evento-clave") (Aste Leiva, 2020). La historización del proceso de creación y crisis del orden entre los dos acontecimientos principales (el golpe militar de 1973 y el contragolpe en forma de estallido social de 2019), es un ejercicio fundamental para comprender el flujo de la energía social actual y las posibilidades de encauzar ritual e institucionalmente las fuerzas disruptivas.

A continuación, presentamos los eventos más relevantes del orden social instaurado en dictadura y continuado durante la transición. Ponemos énfasis en los hechos que agudizaron las fracturas del orden. En la tercera parte y final del texto, reflexionamos en torno a las expectativas de la sociedad chilena que han sido depositadas en la Convención Constitucional y los desafíos que éstas implican. 


\section{EL ORDEN FUNDADO EN DICTADURA, SU CONTINUACIÓN TRANSICIONAL, Y SU CRISIS}

\subsection{Instauración y permanencia del orden como legítimo (1973-2011)}

El golpe de las Fuerzas Armadas en Chile en 1973 operó como momento de escape trascendental del lenguaje y la política (Walder, 2004). La dictadura (1973-1990), que hasta el final violó los derechos humanos, deteniendo ilegalmente, torturando y asesinando, construyó un modelo económico importado desde la Escuela de Chicago. Ello en nombre de la lucha contra la supuesta injerencia extranjera comunista que operaba en el gobierno de Salvador Allende (1970-1973). Así se refundó el orden social en Chile, encarnado en la Constitución de 1980. Redactada por Jaime Guzmán, un ultraconservador católico, franquista, crítico del liberalismo, y fanático anticomunista, fue una pieza extraordinaria de construcción y defensa del libremercado más radical. Guzmán diseñó un sistema donde la vía electoral fuera inútil. No es una interpretación, lo dijo explícitamente (Cristi, 2011). La Constitución la escribió recogiendo conceptos de encíclicas papales de inicios del siglo XX y construyendo una elite política nueva, vinculada a las congregaciones religiosas de alta sociedad (Opus Dei, Legionarios de Cristo, Schoenstat, entre otras), todo en medio de un escenario mundial favorable: Juan Pablo II, el anticomunista, llegaba al poder en 1978 como jefe del Estado Vaticano. Y luego Margaret Thatcher y Ronald Reagan. El mundo se hacía crecientemente amable al proceso (Santoni \& Elgueta, 2018). El experimento chileno empezaba a tomar forma. El Nobel a Milton Friedman en 1976 confirmaba también el camino. Al economista le dieron el premio un año después de visitar a Pinochet (visitó a Augusto Pinochet en 1975), quien ya era una figura mundial de catastrófico prestigio por las violaciones a los Derechos Humanos y los importantes atentados que organizó fuera de Chile contra destacados líderes chilenos (Yaitul Stormansan, 2011). Por la misma época Jorge Luis Borges, el genio literario argentino, también visitó a Pinochet, y no ganó el Nobel (Vincent, 2009). Con Friedman no fue igual. El sistema financiero sueco y europeo veían con buenos ojos el avance de la doctrina del profesor de Chicago.

El símbolo del desarrollo chileno se dio en los llamados 'grupos económicos', articulaciones de ejecutivos e inversionistas que habían tomado control de grandes pedazos del mercado chileno, recorriendo desde la banca a la agricultura, desde el vino a la pesca, desde la minería a los supermercados (Undurraga, 2016). El sostén de su estructura operacional estaba en el sistema de pensiones chileno, un sistema privatizado y administrado por empresas con fines de lucro que generaban una pensión de acuerdo al ahorro y la rentabilidad obtenida para cada trabajador que hubiera pagado un $10 \%$ de su ingreso mensual para tales fines. Ello implicaba que el $10 \%$ de todos los salarios llegaban como dinero fresco cada fin de mes al sistema de pensiones y ese dinero debía colocarse en bolsa. Prácticamente todo ese dinero iba a la Bolsa de Valores de Santiago, fortaleciéndola notoriamente. Las administradoras de las pensiones eran normalmente inversionistas clave en todas las áreas de la economía, administrados por solo cinco o seis empresas (cada una manejando cifras cercanas al presupuesto del país) (Monckeberg, 2015). Las empresas donde se podía invertir estaban predefinidas por la misma estructura de elite. No eran muchas. Ese dinero no fortaleció toda la economía ni a todos los empresarios. El sistema gozaba de una notoria estructura de preferencias. De hecho, cuando el sistema de pensiones fue creado, el dictador Pinochet no estuvo de acuerdo, después de todo era en realidad más fascista que libremercadista. Señaló que un sistema de pensiones de ese tipo concentraría mucho dinero y poder entre poco más de cinco grupos económicos. Este comentario está en las actas oficiales de las reuniones con el creador del modelo de pensiones, José Piñera, hermano de quien llegaría a ser dos veces Presidente de la República, Sebastián Piñera (Matus, 2017).

La inserción de políticas neoliberales en la dictadura vivió un período de inestabilidad y crisis (entre 1973 y 1982) y otro de gloria (entre 1986 y 2013). Este último período tuvo lugar mayormente durante el proceso de transición política a la democracia (1990-2019). A fines de los noventa se consideraba el caso chileno no solo un ejemplo, sino incluso un emblema de una ruta de salida exitosa de regímenes autoritarios, una ruta por lo demás estable y pragmática. Si a esto se sumaba el indiscutible crecimiento económico desde 1986, Chile se convertía en un insoslayable "caso de éxito" a nivel mundial (Cleary, 2007; Ffrench-Davis, 2016; Garretón, 1991; Saavedra, 2014; Siavelis, 2009). La política y la economía evolucionaban en la ruta que se entendía "correcta", esa ruta que había llegado a concebirse en los noventa como "el fin de la historia" (Fukuyama, 1990). 
La consolidación que gozó el neoliberalismo en Chile fue sorprendente. Un modelo puede ser exitoso en su aprobación, pero no es fácil que ocurra con un modelo que básicamente sostenía los siguientes principios (Jara Villarroel, 2016; Kuhlmann, 2018; Stein, 2017):

- Pequeño tamaño del Estado y, con ello, austeridad en el gasto público.

- Programas de apoyo focalizado.

- Mercantilización de servicios sociales (educación, salud y pensiones están a cargo de proveedores privados).

- Estado subsidiario que solo ingresa ante fallas de mercado en la provisión de bienes públicos.

- Prioridad de soluciones privadas a los problemas públicos.

A inicios del siglo XXI los desafíos eran: ¿lograría Chile reducir la desigualdad? ¿lograría consolidar y profundizar el proceso de democratización? ¿lograría dar un salto al desarrollo? (Programa de Naciones Unidas para el Desarrollo, 2000). En medio de esas preguntas, hubo un pequeño aviso, un síntoma de que el éxito chileno quizás no era para tanto, quizás necesitaba ser puesto en cuestión. La "revolución de los pingüinos", movilización de escolares quinceañeros que no distinguían izquierda o derecha, puso en aprietos a la recién electa Presidenta Michelle Bachelet en el año 2006. Pero el sistema político logró afrontar la crisis y resolverla con un pacto transversal de la elite política (la firma del "Acuerdo por la calidad de la educación" y posterior tramitación de la Ley General de Educación o LGE) (Aguilera, 2009; Garretón et al., 2011). Demostraba así su fortaleza la elite transicional: ante los problemas que podían afectar a todos, actuaban como un solo cuerpo.

En ese mismo gobierno (2006-2010), Michelle Bachelet aumentó la protección social, pero ello no implicó un cambio de modelo, sino más bien un aggiornamento (Ezcurra, 1998) de un modelo que buscaba un rostro más humano.

Para el 2010 se eligió como Presidente de la República a uno de los billonarios más poderosos de Chile. Se le conocía como un inversionista impúdico, veloz, inescrupuloso e insensible. Y políticamente había sido muy pragmático. Era el único político relevante de la derecha que no había votado a favor de Pinochet en el Plebiscito de 1988. Eso le abrió un camino porque lo situaba en una derecha democrática que no apoyaba a Pinochet, fortaleciendo una especie de liberalismo genuino, no solo económico. Su triunfo en 2010 marcaba la legitima- ción política y social de las políticas neoliberales y del empresariado financiero como figura histórica central del Chile contemporáneo (Castiglioni, 2010; Nercesian \& Mendoza, 2021; Undurraga, 2012). Aquel primer año de gobierno permitió a Sebastián Piñera conocer cifras de aprobación sobre el $60 \%$ (gracias al rescate de los 33 mineros atrapados en la mina San José) (Mella Polanco, 2012).

La penetración cultural y política del modelo neoliberal parecía definitiva.

\subsection{Crisis del orden (2011)}

La era dorada del neoliberalismo chileno llevaba décadas en lo económico, pero con la llegada de Piñera al gobierno había dado un paso más (de hecho, el paso más importante): seducía el alma del pueblo y permitía elegir un presidente que simbolizaba a los principales millonarios de Chile.

¿Eran los pilares realmente sólidos? Las políticas de austeridad y privatización habían transformado los derechos sociales en una excepción y la provisión de esos servicios sociales estaba fuertemente privatizada: salud, educación y pensiones tenían amplísima (o total) provisión privada (Espinoza, 2017; González Pizarro, 2020; Goyenechea \& Sinclaire, 2013; Ortiz et al., 2019). Las demandas de mejor calidad de vida desde el punto de vista de la protección empezaron a ser un problema para el modelo de sociedad que no podía resolver las sucesivas demandas sin sacrificar parte de sus políticas. Ya no bastaba, en tanto satisfactor o placebo, la expectativa del acceso a bienes y la sensación de un futuro mejor para la siguiente generación. Comenzaba a exigirse un presente que otorgase derechos. Sin embargo, estas demandas no podían ser procesadas. La Constitución chilena era una pieza briIlante que resumía el pacto intraderecha que había implicado la dictadura: neoliberalismo económico, fascismo político (Cristi, 2011). Con la redacción de la Constitución de 1980, en Chile se construyeron no solo pilares del modelo, sino además barreras para evitar que la participación política y la deliberación pública pudieran hacer algo contra esos pilares: el Tribunal Constitucional operaba como una institución orientada a evitar los cambios en la sociedad (Bassa Mercado, 2015; Soto Delgado, 2018). Esto explica que fuera imposible la realización de cualquier demanda social.

Diversas investigaciones ya manifestaban la existencia de niveles de malestar llamativos desde hacía décadas y la desigualdad seguía siendo un flagelo no resuelto (Mayol, 2012a; Mira, 2011; Progra- 
ma de Naciones Unidas para el Desarrollo, 1998; Ruiz Encina, 2007). La ciudadanía confiaba en que las falencias experimentadas se resolverían dentro del mismo modelo de sociedad, pero las dudas arreciaron en el año 2011. Y las trizaduras anteriores se tornaron fracturas. $Y$ en algún momento las fracturas parecían amenazar la estructura general. Dos grandes fantasmas del neoliberalismo se unieron, sus más tradicionales puntos débiles: los derechos sociales postergados por las políticas de austeridad, y las tendencias oligopólicas del mercado. Si ya Chile era uno de los países más oligárquicos de la historia latinoamericana, la consolidación oligopólica de los llamados 'grupos económicos' situaba el escenario en un punto con elevado potencial crítico.

Decíamos que este era el primer fantasma: los derechos sociales postergados. Un Estado débil no era capaz de generar protección social, y la ilusión de una mejor calidad de vida solo basada en el crecimiento del ingreso (promedio) y en el acceso al crédito comenzaba a ver sus límites. Pero había un segundo fantasma que habitaba en el corazón de la promesa: el oligopolio. 0 , dicho de otra forma, la falsedad última de la economía de mercado. Los chilenos habían aceptado la existencia de fallas de mercado, pero en un par de años la prestigiosa estructura de grandes empresas que ofertaban sus servicios en el marco de la competencia devino en una imagen muy distinta. El mercado chileno fue develando sus desequilibrios, injusticias y arbitrariedades con mucha intensidad y en poco tiempo. El surgimiento vertiginoso y simultáneo de escándalos empresariales que iban desde la colusión, el tráfico de influencias, el financiamiento ilegal de autoridades políticas (a lo que nunca se le llamó corrupción), los casos de abusos por acciones unilaterales en el mundo financiero (cambio de contratos sin aprobación del cliente) ante el desconocimiento de los clientes, el cobro de intereses escandalosos por deudas educacionales que a su vez estaban garantizadas por el Estado, en fin, todos estos casos, que arreciaron desde 2011 a 2015, dejaron al modelo económico sin posibilidad de sostener su promesa (Arévalo Mellado \& Soto Leiva, 2014; Donoso \& López, 2016; Gamboa \& Segovia, 2016; Mayol \& Contreras, 2015).

Todo proyecto de sociedad es político (aunque el neoliberalismo pretenda ser apolítico). Y la política requiere siempre de confianza y legitimidad. Este proyecto de sociedad, el neoliberalismo, se fundamentaba en la confianza en el mercado. Los empresarios no serían un dechado de virtudes, pero al menos serían fieles a las reglas del mercado: com- petirían. No serían sensibles a los problemas de la gente, nadie esperaba eso. Pero no traicionarían la competencia, cada uno debía ir por lo suyo. La colusión del papel higiénico fue un símbolo de la locura: resultaba que, entre una enorme cantidad de marcas de papel higiénico, solo había una que no estaba participando en un esquema de colusión que había implicado utilidades extra de más de US $\$ 1000$ millones. Y había ocurrido lo mismo con las farmacias, los pollos, los refrigeradores, empresas navieras y pañales. Por otro lado, diversas empresas habían modificado contratos unilateralmente (Arévalo Mellado \& Soto Leiva, 2014; Donoso \& López, 2016; Gamboa \& Segovia, 2016).

La explosión social de 2011 en Chile abrió el ciclo de crisis. La fisura de 2006 se había convertido en fractura. Casos emblemáticos medioambientales, una nueva crisis de la educación y el descubrimiento de un esquema crediticio abusivo por parte de una empresa de retail, que había afectado a ochocientas mil personas, cultivaron el primer momento de crisis expresa del modelo y su legitimidad. Las universidades privadas en Chile habían proliferado, pero no podían obtener rentas, eran sin fines del lucro. Sin embargo, se vendían en cientos de millones de dólares. ¿Por qué? Porque lucraban. Y claro, estaba prohibido lucrar, pero no tenía sanción. En 2011 la palabra 'lucro' dejó de significar rentabilidad y pasó a significar abuso (Mayol, 2012b).

Durante el año 2011 se 'politizó el malestar', esto es, se modificó la forma social del malestar al sacarlo de la anomia e insertarlo en la política. Se requería una respuesta política. Y existió.

El segundo gobierno de Michelle Bachelet (20142018), con una nueva coalición que contaba en su interior con los dos principales dirigentes estudiantiles del 2011, sería el primer esfuerzo de respuesta (Palacios-Valladares \& Ondetti, 2019). No funcionó. Gran parte del problema radicaba en haber sectorizado la crisis, asumiendo que ella radicaba fundamentalmente en educación. Los cuestionamientos por parte de la ciudadanía podían tener uno o más objetos, pero la crítica de fondo involucraba a todo el modelo de sociedad y fundamentalmente a la interacción ya ilegítima entre modelo económico y expectativas políticas. La evidencia de esta profundidad fue quedando clara durante el segundo gobierno de Bachelet, uno profundamente errático. Grandes movilizaciones retornaron en 2016 con el foco en la crítica al sistema de pensiones privatizado (Rozas Bugueño \& Maillet, 2019; Sola-Morales, 2020).

Para el 2018, nuevamente se eligió a Sebastián Piñera para llevar el gobierno. Convencido de 
que esta vez la consolidación del modelo sí llegaba, avanzó por su camino. El sistema político y la elite empresarial no comprendieron la profundidad del problema. La convicción de la derecha neoliberal fue que el "terremoto social" había desaparecido del horizonte al haberse elegido nuevamente a Sebastián Piñera. Pero ese diagnóstico se reveló equivocado. La legitimidad se había destruido. Era un evidente punto de no retorno. Piñera tuvo que lidiar con el auge del movimiento feminista en 2018 (Contreras, 2021; Hiner et al., 2021; Ponce Lara, 2020; Reyes-Housholder \& Roque, 2019). Y 19 meses después de iniciado su gobierno, de un día para otro, ocurrió lo impensado: la sociedad de mercado, la elite chilena, la Constitución de 1980, los grupos económicos, se quedaron sin poder en 24 horas. Se puede resumir de forma simple: Sebastián Piñera dijo que Chile era un oasis a comienzos de octubre de 2019 y diez días después (18 de octubre) una protesta pequeña contra el aumento de la tarifa del Metro de Santiago derivó en un estallido social con saqueos, quema de infraestructura y con daños a la propiedad pública y privada de alrededor de US\$4500 millones en un fin de semana. Confundido, el Presidente de la República declaró una guerra a nadie, mientras los militares señalaban que ellos no irían a esa guerra (Navarro \& Tromben, 2019; Spyer Dulci \& Alvarado Sadivia, 2021; Verdes-Montenegro, 2021). El ya referido e histórico estallido social no solo desdibujó los límites del orden (como ocurrió con las movilizaciones del 2011), sino que prácticamente los desintegró.

Chile tendría que suspender la COP25, la APEC, la final de la Copa Libertadores de América, tres eventos clave para el gobierno porque marcaban el liderazgo político de Chile en Sudamérica (Mayol, 2020).

\section{LOS DESAFÍOS DE LA CONVENCIÓN CONSTITUCIONAL}

Desde el estallido social de octubre de 2019 la elite política chilena se ha enfrentado al desafío de cómo recuperar algo de legitimidad y capacidad gubernativa. Casi un mes después, en noviembre de 2019, con protestas diarias, jornadas laborales reducidas en horarios en la mayor parte de las ciudades del país, y graves situaciones de violaciones a los derechos humanos por parte de la policía chilena, la elite se preparaba para una semana terrorífica: el cumplimiento del primer mes del estallido (el día 18 de noviembre). Muchos inversionistas se dolarizaban y el
Banco Central debía pedir un crédito de US\$20.000 millones para poder afrontar la defensa del valor de la moneda. Y esa misma semana se cumplía, para colmo, el aniversario de un año de un asesinato de Camilo Catrillanca a manos de Carabineros. Aterrorizados, en el gobierno vislumbraban la posibilidad de una crisis total. El 12 de noviembre de 2019 el gobierno de Sebastián Piñera decidió proponer un cambio constitucional por la vía participativa (Ramírez et al., 2020). Fue así como un gobierno de derecha entregaba a una asamblea constituyente la posibilidad de redactar una nueva constitución. Y asumía la pérdida de un recurso muy valioso.

Desde entonces el proceso constituyente ha tenido algunos trastornos ocasionados por la pandemia mundial, pero ha seguido su curso. El sistema de partidos prácticamente no ha logrado tener control del proceso, mientras que el gobierno no ha tenido ninguno. El 25 de octubre de 2020 (es decir, un año después del estallido social) se realizó el Plebiscito Nacional de 2020 ('plebiscito de entrada'), orientado a aprobar la idea de cambio constitucional y el método de asamblea para ello (el plebiscitó contempló dos preguntas: ‘¿Quiere usted una nueva Constitución?’ y ‘¿Qué tipo de órgano debiera redactar la nueva Constitución?'). El resultado fue una derrota gigante para los sectores conservadores: $80 \%$ de la población votó 'Apruebo' por el cambio constitucional y la misma proporción eligió 'Convención Constitucional', un método que elegiría a todos los representantes para la asamblea en una elección especial sin contemplar la inserción de parlamentarios vigentes en el Congreso Nacional. Estos resultados reflejaban una clara polarización entre la ciudadanía versus la elite (o una tensión de clase social, empleando terminología del materialismo histórico) (Arancibia-Bustos et al., 2021). Luego se negoció el proceso electoral para convencionales, lo que determinó una nueva derrota de la elite política: se podrían construir listas de independientes, habría paridad de género (por primera vez en la historia de una elección constituyente a nivel mundial) y habría cupos para pueblos originarios (Suárez-Cao et al., 2021). La elección marcó una nueva derrota para la derecha chilena: necesitaban el 33\% (52 de 155 escaños) de convencionales electos para vetar artículos en el proceso de redacción de la nueva constitución, pero obtuvieron algo por sobre el 20\% (37 de 155 escaños).

En julio de 2021 comenzó el funcionamiento de la Convención Constitucional (el nombre no incluye la palabra 'Asamblea', lo que fue una concesión formal a la derecha, que no quería el rótulo ‘Asamblea 
Constituyente' usado tradicionalmente por la izquierda). El proceso terminará necesariamente antes de doce meses desde el inicio de su funcionamiento (julio de 2022). Por entonces se habrá elegido un nuevo gobierno y un nuevo Congreso Nacional en Chile. Y en agosto o septiembre de 2022 se realizará un nuevo plebiscito (conocido como el 'Plebiscito de salida') que aprobará o rechazará la propuesta del texto constitucional. Todo este proceso pretende revitalizar la legitimidad de un orden político que no está siendo capaz de vertebrar respuestas simbólicas y de política pública. Sin embargo, la herramienta utilizada, no obstante ser de carácter refundacional, parece ser todavía insuficiente como repertorio de acción para catalizar y conducir el proceso de transformación política y social del nuevo Chile. ¿Por qué? El estallido social de 2019 ha requerido una búsqueda de una contención narrativa que otorgue sentido al proceso y permita configurar una propuesta de orden social futuro. Ese proceso se inició en forma de protesta durante el mes posterior al 18 de octubre de 2019, generando una gran cantidad de nuevas significaciones en las calles, ya sea en forma de grafitis o de textos de protesta, cambio de nombres de monumentos o emplazamientos, en fin. Luego del acuerdo del 15 de noviembre, donde nace el proceso constituyente formalmente, dicha construcción semántica quedó a cargo de la construcción de la Constitución Política. A la vista de una ausencia radical de modificaciones sistémicas en otros ámbitos, todos los huevos se pusieron en la misma canasta (de forma similar a cómo Laclau se refiere a las cadenas de equivalencias (Laclau, 2006; Retamozo, 2017)). El proceso requiere resolver desafíos en cuatro dimensiones:

- transformar el modelo económico

- transformar el modelo de Estado

- renovar la elite política

- redactar una nueva constitución política

Todas estas son semánticas esenciales para la configuración del nuevo orden. Sin embargo, hay solo una herramienta: la Convención Constitucional. La capacidad de esa herramienta para generar todo el volumen de contenidos indispensables parece discreta.

Podría pensarse que la Lista del Pueblo (agrupación que obtuvo 27 escaños en la Convención Constitucional y que aspira a participar en las elecciones parlamentarias y presidenciales de 2021), que reivindica su carácter popular y su vinculación con el estallido social, es un esfuerzo ciudadano por renovar la elite política. Sin embargo, hasta la fecha (agosto de 2021) han demostrado tener severos problemas de orgánica: nueve constituyentes han abandonado la colectividad o han congelado su participación en ella, y las dificultades en la definición de un candidato presidencial podría fraccionar a la Lista del Pueblo.

Por otra parte, la transformación del modelo económico y el Estado no es algo que dependa enteramente de la Constitución, sino que se requiere del apoyo del poder Ejecutivo y el Legislativo en lo que, insistimos, efectivamente se trata de una refundación de Chile. Aquí, por supuesto, adquieren muchísima relevancia las elecciones parlamentarias y presidenciales de noviembre de 2021. Y mencionar las elecciones no es asunto baladí, porque existe otra variable: la débil participación ciudadana en los asuntos públicos, reducida meramente a una participación electoral que disminuye con el paso del tiempo (Morales Quiroga, 2020). ¿Serán capaces los partidos políticos de servir como plataforma de representación política en medio de un contexto en que las instituciones carecen de legitimidad?

Aunque existan conclusiones obvias (aumentar los derechos sociales, acabar con la discriminación institucionalizada, y generar mejores mecanismos de participación ciudadana), eso no es suficiente parar configurar un proyecto.

Marx pensó que la acumulación de capital antes del capitalismo fue clave para que dicho orden surgiera (acumulación originaria) (Marx, 1867/1976). Weber complementó esto señalando que el proceso de acumulación necesitaba racionalización para convertir la energía dispersa en sistema (Weber, 1905/2011). Esto solo era posible mediante una imagen de mundo coherente e inteligible culturalmente. La acumulación necesita tener un sentido. Estas consideraciones nos muestran un déficit del proceso chileno: una enorme descarga de energía disruptiva ha trastocado los cimientos del orden social fundado en dictadura, pero se carece de un proceso de acumulación de sentido suficientemente intenso capaz de generar las condiciones para controlar, sublimar y procesar dicha energía.

\section{REFERENCIAS}

Aguilera, C. (2009). Un análisis de las comisiones asesoras presidenciales del gobierno de MicheIle Bachelet (Documento de trabajo Programa de Gobernabilidad). FLACSO Chile.

Arancibia-Bustos, Á., Flores-Capriles, J. y Gutiérrez-Peñaloza, S. (2021). Del estallido a la lucha de clases. Claves para el análisis de los resultados del plebiscito por una nueva constitución en 
Chile. Desde el Sur, 13(1), e0004. https://doi. org/10.21142/DES-1301-2021-0004

Araya, J., Azócar, C., Azócar, C. y Mayol, A. (2019). Exploring the daily life of mining communities: The case of Antofagasta, Chile. Rural Society, 28(3), 226-239. https://doi.org/10.1080/10371656. 2019.1678801

Arévalo Mellado, S. y Soto Leiva, C. (2014). Fraudes financieros y contables en Chile en los últimos diez años [Tesis de pregrado, Universidad del Bío-Bío]. Repositorio Digital del Sistema de Bibliotecas Universidad del Bio-Bio. http://repobib.ubiobio.cl/jspui/handle/123456789/3051

Aste Leiva, B. (2020). Estallido social en Chile: La persistencia de la Constitución neoliberal como problema. DPCE Online, 42(1), 3-19.

Bassa Mercado, J. (2015). El Tribunal Constitucional en la Constitución chilena vigente. En J. Bassa Mercado, J. C. Ferrada Bórquez y C. Viera Álvarez (eds.). La Constitución Chilena (pp. 253-284). Lom Ediciones.

CAStiglioni, R. (2010). Chile y el giro electoral: "la vergüenza de haber sido y el dolor de ya no ser". Revista de Ciencia Política, 30(2). https://doi. org/10.4067/S0718-090X2010000200004

Cleary, E. (2007). Chile: Entre la imagen de éxito y los fantasmas del subdesarrollo. Polis, 18. https:// doi.org/10.32735/S0718-6568/2007-N18-546

Contreras, M. J. (2021). Poniendo los cuerpos en la lucha contra la violencia basada en el género: El Tsunami Feminista en Chile el 2018. Taller de Letras, 68, 162-180. https://doi.org/10.7764/ t|68162-180

CRISTI, R. (2011). El pensamiento político de Jaime Guzmán. Lom Ediciones.

Delamaza, G., Thayer Correa, L. E. y Gaete, J. M. (2015). Diferencias territoriales en las percepciones políticas en Chile: Una aproximación operacional a la escala nacional y regional. Si Somos Americanos, 15(1), 15-48. https://doi.org/10.4067/ S0719-09482015000100002

Donoso, V., y LóPEZ, F. (2016). Escándalos corporativos en Chile: Impacto en los precios accionarios de las empresas involucradas. Gestión y Tendencias, 1(2), 2-6. https://doi.org/10.11565/ gesten.v2i1.10

Espinoza, O. (2017). Privatización de la educación superior en Chile: Consecuencias y lecciones aprendidas. EccoS, 44, 175-202. https://doi. org/10.5585/eccos.n44.8070

EzcuRRA, A. M. (1998). El neoliberalismo frente a la pobreza mundial. Ediciones Abya-Yala.

Ffrench-Davis, R. (2016). Progresos y retrocesos del desarrollo económico de Chile en los gobiernos de la Concertación: 1990-2009. El Trimestre Económico, 83(329), 5-34. https://doi. org/10.20430/ete.v83i329.190

FleEt, N. (2011). Movimiento estudiantil y transformaciones sociales en Chile: Una perspectiva sociológica. Polis, 30. https://doi.org/10.4067/ S0718-65682011000300005

Fukuyama, F. (1990). ¿El fin de la historia? Estudios Públicos, 37, 5-31.

Gaete Lagos, J. (2010). Andrés Baeza, Andrés Estefane, Juan Luis Ossa, Joaquín Fernández, Cristóbal García-Huidobro, Nicolás Ocaranza y Pablo Moscoso, XX Historias del siglo veinte chileno, Santiago, Ediciones B Chile S.A, 2008, 1era edición, 492 p. Polis, 9(26). https://doi.org/10.4067/ S0718-65682010000200023

Gaitán Bayona, J. L. (2015). Una risa en el desierto: El arte de la resurrección y sermones y prédicas del Cristo de Elqui. La Palabra, 27, 159-170. https://doi.org/10.19053/01218530.4002

Gamboa, R., y Segovia, C. (2016). Chile 2015: Falla política, desconfianza y reforma. Revista de Ciencia Política, 36(1), 123-144. http://dx.doi. org/10.4067/S0718-090X2016000100006

GARREtóN, M. A. (1991). La redemocratización política en Chile. Transición, inauguración y evolución. Estudios Públicos, 42, 101-133.

Garretón, M. A., Cruz, M. A., Aguirre, F., Bro, N., Farías, E., Ferreti, P. y Ramos, T. (2011). Movimiento social, nuevas formas de hacer política y enclaves autoritarios: Los debates del Consejo Asesor para la Educación en el gobierno de Michelle Bachelet en Chile. Polis (Santiago), 30. https://doi. org/10.4067/S0718-65682011000300006

González Pizarro, M. A. (2020). Privatización de pensiones, el caso chileno: Historia, resultados y perspectivas [Tesis doctoral, Universidad de Salamanca]. Gestión del Repositorio Documental de la Universidad de Salamanca (GREDOS). https://doi.org/10.14201/gredos.145296

GOYENECHEA, M. y Sinclaire, D. (2013). La privatización de la salud en Chile. Políticas Públicas, 6(1), 35-52.

Hiner, H., López, A. y Badilla, M. (2021). ¿El neoliberalismo nace y muere en Chile? Reflexiones sobre el 18-0 desde perspectivas feministas. História Unisinos, 25(2). https://doi.org/10.4013/ hist.2021.252.07

Jamison, A. (2010). Climate change knowledge and social movement theory. WIREs Climate Change, 1(6), 811-823. https://doi.org/10.1002/wcc.88 Jara Villarroel, N. J. (2016). ¿Democracia sustantiva 
con Estado Mínimo? Los Derechos Sociales en el Neoliberalismo [Tesis de Magíster, Universidad de Chile]. Repositorio académico de la Universidad de Chile. http://repositorio.uchile.cl/ handle/2250/180826

Kunlmann, C. (2018). Endeudamiento en Chile. La deuda como dispositivo de la gubernamentalidad neoliberal [Tesis de pregrado, Universidad de Chile]. Repositorio académico de la Universidad de Chile. http://repositorio.uchile.cl/handle/2250/159204

LACLAU, E. (2006). La razón populista (1. ed). Fondo de Cultura Económica.

LATTA, A. (2011). Los desastres planificados: Megaproyectos y trauma socio-ambiental, el caso de HidroAysén. Sociedad Hoy, 20, 111-129.

Marx, K. (1976). Capital: A Critique of Political Economy: Vol. Volume 1 (B. Fowkes, Trad.). Penguin Books. (Originalmente publicado en inglés en 1867)

Matus, A. (2017). Mitos y verdades de las AFP. Editorial Aguilar.

Mayol, A. (2012a). El derrumbe del modelo: La crisis de la economía de mercado en el Chile contemporáneo (Primera edición). LOM Ediciones.

MaYol, A. (2012b). No al lucro: De la crisis del modelo a la nueva era política. Editorial Debate.

Mayol, A. (2019). Big bang. Estallido social 2019. Modelo derrumbado-Sociedad rota-Política inútil. Editorial Catalonia.

Mayol, A. (2020). Protestas y Disrupción Política y Social en Chile 2019: Crisis de Legitimidad del Modelo Neoliberal y Posible Salida Política por Acuerdo de Cambio Constitucional. Asian Journal of Latin American Studies, 33(2), 85-98.

MaYol, A. y ContreRas, P. (2015). Un fantasma recorre el mercado: ¿Es El Caso La Polar el espíritu del modelo? En A. Garrido Núñez, F. González Silva e I. Torres Zapata (eds.). Regulación Económica: Caso La Polar. Compilación de Ensayos Multidisciplinarios (pp. 49-58). Editorial USACH.

Mayol Miranda, A. y Azócar Rosenkranz, C. (2011). Politización del malestar, movilización social y transformación ideológica: El caso "Chile 2011". Polis, 30. https://doi.org/10.4067/S071865682011000300008

Mella Polanco, M. (2012). Efectos sociales del terremoto en Chile y gestión política de la reconstrucción durante el gobierno de Sebastián Piñera (2010-2011). Enfoques, 10(16), 19-46.

MIRA, A. (2011). Crisis de representatividad y estallido social: Una aproximación a la actual experiencia chilena. Polis, 30. https://doi.org/10.4067/ S0718-65682011000300009
MonckeberG, M. O. (2015). El saqueo de los grupos economicos al estado de Chile. Debolsillo.

Morales Quiroga, M. (2020). Estallido social en Chile 2019: Participación, representación, confianza institucional y escándalos públicos. Análisis Político, 33(98), 3-25. https://doi.org/10.15446/ anpol.v33n98.89407

Navarro, F. y Tromben, C. (2019). "Estamos en guerra contra un enemigo poderoso, implacable": Los discursos de Sebastián Piñera y la revuelta popular en Chile. Literatura y Lingüística, 40, 295-324. http://dx.doi.org/10.29344/0717621x.40.2083

NerCESiAn, I.y MendozA, M. (2021). Empresariadoy poder político: PPK y Piñera (2010-2018). Revista Mexicana de Sociologia, 83(1), 73-102. https://doi. org/10.22201/iis.01882503p.2021.1.60023

Ortiz, l., Durán-Valverde, F., Urban, S., Wodsak, V. y Yu, Z. (2019). La privatización de las pensiones: Tres décadas de fracasos. El Trimestre Económico, 86(3), 799-838. https://doi.org/10.20430/ete. v86i343.926

Palacios-Valladares, I. y Ondetti, G. (2019). Student Protest and the Nueva Mayoría Reforms in Chile. Bulletin of Latin American Research, 38(5), 638-653. https://doi.org/10.1111/blar.12886

Penagla Vásquez, F. E. y Valenzuela Van Treek, E. (2014). Rebeldía en Calama: Desafío al orden centralista chileno en un contexto de boom minero. Revista Mexicana de Ciencias Políticas y Sociales, 59, 161-185.

Ponce Lara, C. (2020). El movimiento feminista estudiantil chileno de 2018: Continuidades y rupturas entre feminismos y olas globales. Izquierdas, 49, 1554-1570. http://dx.doi.org/10.4067/s071850492020000100280

Programa de Naciones Unidas para el Desarrollo. (1998). Desarrollo Humano en Chile 1998: Las paradojas de la modernización.

Programa de Naciones Unidas para el Desarrollo. (2000). Desarrollo humano en Chile 2000: Más sociedad para gobernar el futuro. https://www. cl.undp.org/content/chile/es/home/library/human_development/mas-sociedad-para-gobernar-el-futuro.html

Ramírez, C., Yañez-Urbina, C. y Salinas, I. (2020). Chile, la democracia se acabó: Crisis institucional en el 18-0 chileno. Re-presentaciones. Periodismo, Comunicación y Sociedad, 12, 6-28. https:// doi.org/10.35588/rp.v0i12.4317

Retamozo, M. (2017). La teoría del populismo de Ernesto Laclau: Una introducción. Estudios Políticos, 41, 157-184. https://doi.org/10.1016/j. espol.2017.02.002 
Reyes Herrera, S. E. y Rodríguez Torrent, J. C. (2015). Proyecto Hidroaysén: Capitalismo extractivista, regulación estatal y acción colectiva en la Patagonia. Polis, 40. https://doi.org/10.4067/ S0718-65682015000100021

Reyes-Housholder, C. y Roque, B. (2019). Chile 2018: Desafíos al poder de género desde la calle hasta La Moneda. Revista de Ciencia Política, 39(2), 191-215. https://doi.org/10.4067/S0718090X2019000200191

Romero Toledo, H. (2014). Ecología política y represas: Elementos para el análisis del Proyecto HidroAysén en la Patagonia chilena. Revista de geografía Norte Grande, 57, 161-175. https://doi. org/10.4067/S0718-34022014000100011

Rozas Bugueño, J. y Maillet, A. (2019). Entre marchas, plebiscitos e iniciativas de ley: Innovación en el repertorio de estrategias del movimiento No Más AFP en Chile (2014-2018). Izquierdas, 48, 1-21. https://doi.org/10.4067/S071850492019000400001

Ruiz Encina, C. (2007). Actores sociales y transformación de la estructura social. Revista de Sociología, 21, 209-233. https://doi.org/10.5354/0719-529X.2007.27525

SaAvedra, E. (2014). El modelo económico-político de Chile: Desarrollo institucional en la encrucijada. Economía y Política, 1(1), 115-146. https://doi. org/10.15691/07194714.2014.004

Santoni, A. y Elgueta, R. (2018). "Chile viene de vuelta". El gremialismo, la síntesis conservadora-neoliberal y la crisis del occidente europeo (1980-89). Cuadernos de historia, 48, 161-185. https://doi. org/10.4067/S0719-12432018000100161

Siavelis, P. (2009). Enclaves de la transición y democracia chilena. Revista de Ciencia Política 29(1), 3-21. https://doi.org/10.4067/S0718 090X2009000100001

Sola-Morales, S. (2020). No + AFP: Videoactivismo, movilización ciudadana y protestas por unas pensiones dignas en el Chile neoliberal. Comunicación y Medios, 41, 14-28. https://doi. org/10.5354/0719-1529.2020.55907

Sото Delgado, P. (2018). El giro conservador en torno a las sanciones administrativas por obra del Tribunal Constitucional en Chile: Transformando a la Administración en juez para desproteger el interés público. Revista de la Facultad de Derecho, 45, e20184509. http://dx.doi.org/10.22187/rfd2018n45a9

Spyer Dulci, T. M. y Alvarado Sadivia, V. (2021). El Estallido Social en Chile: ¿rumbo a un Nuevo Constitucionalismo? Revista Katálysis, 24(1), 43-52. https:// doi.org/10.1590/1982-0259.2021.e73555

Stein, R. H. (2017). La protección social en América Latina y la particularidad de la asistencia social. SER Social, 19(40), 49-68. https://doi. org/10.26512/ser_social.v19i40.14671

Suárez-Cao, J., Arce-Riffo, J., Riquelme Parra, S., Acevedo de la Harpe, C., Espinoza Soto, N., Disi Pavlic, R., Roque, B., Moyano, V. y Vega Elizondo, B. (2021). Los legados de represión y exclusión en Chile ante la oportunidad de la nueva constitución. IBEROAMERICANA. América Latina - España - Portugal, 21(77), 239-270. https://doi. org/10.18441/ibam.21.2021.77.239-270

Tesche RoA, P. (2014). La locura religiosa en La Vuelta del Cristo de Elqui. Atenea (Concepción), 510, 111-126. https://doi.org/10.4067/S071804622014000200008

UndURRAGA, T. (2012). Transformaciones sociales y fuentes de poder del empresariado chileno. Ensayos de Economía, 41, 201-225.

UNDURRAGA, T. (2016). Business, politics and ideology: Neoliberalism and capitalist class formation in Argentina and Chile (1990 - 2014). Revista de Ciencia Política, 54(2), 175-205.

Valenzuela, E., Penaglia, F. y Basaure, L. (2016). Acciones colectivas territoriales en Chile, 2011-2013: De lo ambiental-reivindicativo al autonomismo regionalista. EURE, 42(125), 225-250. https://doi. org/10.4067/S0250-71612016000100010

Verdes-Montenegro, F. J. (2021). Militarización visual en momentos de crisis: Los casos de Lenin Moreno y Sebastián Piñera durante las movilizaciones de octubre de 2019. Relaciones Internacionales, 94(1), 111-130. https://doi. org/10.15359/ri.94-1.5

VinCENT, M. (2009). Jorge Luis Borges: La visión del ámbar. Escritura y Pensamiento, 12(24), 231234. https://doi.org/10.15381/escrypensam. v12i24.7752

WALDER, P. (2004). La palabra circulante. Territorialización económica del lenguaje. Polis, 9. https:// doi.org/10.32735/S0718-6568/2004-N9-336

Weber, M. (2011). La ética protestante y el espíritu del capitalismo. Fondo de Cultura Económica.

Weber, M. (2014). Economía y sociedad. Fondo de Cultura Económica.

Yaitul Stormansan, J. (2011). Los años del capitalismo renovado: La influencia del Milton Friedman en Chile. Espacio Regional, 2(8), 57-76. 